\title{
A NOTE ON A THEOREM BY WITT ${ }^{1}$
}

ROBERT M. THRALL

1. Introduction. Let $F$ denote the free group with $n$ generators and let $F^{c}$ be the $c$ th member of the lower central series ${ }^{2}$ of $F$. Witt ${ }^{3}$ has shown that $Q^{c}=F^{c} / F^{c+1}$ is a free abelian group with $\psi_{c}(n)$ $=(1 / c) \sum \mu(c / d) n^{d}$ generators (the summation is over all divisors $d$ of $c$ and $\mu$ is the Möbius $\mu$-function).

The set of $k$ th powers in $F$ generates a normal subgroup $H_{k}$. Let $F_{k}=F / H_{k}$ and $G_{k, c}=F_{k} / F_{k}^{c+1}$. We shall call $F_{k}$ the free $k$-group and $G_{k, c}$ the free k-group of class $c$. It is a consequence of Witt's result that $F_{k}^{c} / F_{\boldsymbol{k}}^{c+1}$, the central of $G_{k, c}$, is abelian and has at most $\psi_{c}(n)$ generators. In this note we show that if $p$ is a prime greater than $c$, and $q=p^{\alpha}$, then the central of $G_{q, c}$ is of order $q^{N}$ where $N=\psi_{c}(n)$. If the prime divisors of $k$ are all greater than $c$, an analogous result holds for the central of $G_{k, c}$ as a consequence of Burnside's theorem that a nilpotent group is the direct product of its Sylow subgroups.

Let $M_{c}$ denote the space of tensors of rank $c$ over the $G F[p]$. A homomorphic mapping of $M_{c}$ upon the central of $G_{p, c}$ is set up and enables one to apply the theory of decompositions of tensor space under the full linear group $\bmod p$, to determine all characteristic subgroups of $G_{p, c}$ which lie in its central. This theory is applied to determine all the characteristic subgroups of $G_{p, c}$ for $c<5$ and a multiplication table is constructed for $G_{p, 3}$.

2. Commutator calculus. ${ }^{4}$ Let $s_{1}, s_{2}, \ldots$ be operators in any group $G$ and set $s_{12}=\left(s_{1}, s_{2}\right)=s_{1}^{-1} s_{2}^{-1} s_{1} s_{2}$ and $s_{12} \ldots k=\left(s_{12} \ldots k-1, s_{k}\right) . s_{12} \ldots k$ is called a simple commutator of weight $k$ in the components $s_{1}, \cdots, s_{k}$. The group $G^{k}$ generated by the simple commutators of weight $k$ for all choices of $s_{1}, \cdots, s_{k}$ in $G$ is called the $k$ th member of the lower central series of $G$. If $s \in G^{k}$ but $s \notin G^{k+1}$, then $s$ is said to have weight $k$ in $G$.

For all $s_{1}, s_{2}, s_{3}$ in $G$ we have

$$
\left(s_{1} s_{2}, s_{3}\right)=s_{13} s_{132} s_{23}, \quad\left(s_{1}, s_{2} s_{3}\right)=s_{13} s_{12} s_{123} .
$$

Let the weight of $s_{i}$ be $\alpha_{i}$ and set $\alpha=\alpha_{1}+\cdots+\alpha_{k}+1$. The following relations are then true:

\footnotetext{
1 Presented to the Society, April 13, 1940.

${ }^{2}$ For definition see $\$ 2$ below or [4, p. 49].

$3[7$, p. 153].

4 The relations in this section are either taken directly from Hall, Magnus, or Witt or are immediate consequences of their theorems. See [4, 6 and 7$]$.
} 


$$
\begin{aligned}
& s_{123} \ldots{ }_{k} s_{213} \ldots k \equiv I \bmod G^{\alpha}, \\
& s_{123} \ldots_{k} s_{231} \ldots{ }_{k} s_{312} \ldots k \equiv I \bmod G^{\alpha} \text {, } \\
& \left(s_{1}^{a_{1}}, s_{2}^{a_{2}}, \cdots, s_{k}^{a_{k}}\right) \equiv\left(s_{12} \ldots k\right)^{a_{1} a_{2} \cdots a_{k}} \bmod G^{\alpha} .
\end{aligned}
$$

If now $\alpha-1=k m, m=\operatorname{minimum}\left(\alpha_{1}, \cdots, \alpha_{k}\right)$ and $\rho_{\beta}=\prod_{\delta=1}^{\delta=n} s_{\delta}^{a_{\beta} \beta}$, $\beta=1, \cdots, k$, it follows that

$$
\rho_{12} \ldots k \equiv \prod_{\beta_{\nu=1}}^{n}\left(s_{\beta_{1}} \cdots \beta_{k}\right)^{a_{1} \beta_{1}} \cdots a_{k \beta_{k}} \bmod G^{\alpha} .
$$

3. The groups $F_{q}$. Let $F$ be the free group generated by $s_{1}, \cdots, s_{n}$, and denote by $\bar{H}_{k}$ the smallest normal subgroup containing the $k$ th powers of all simple commutators of $s_{1}, \cdots, s_{n}$.

Lemma I. Let $q=p^{\alpha}$, $p$ any prime. Then $s^{q} \in \bar{H}_{q} \cup F^{p}$ for any element $s \in F$.

Proof By INDUCtion. The lemma is trivial for $s$ of weight greater than $p-1$. Suppose the lemma true for all weight greater than $c$ and let $s$ be of weight $c$. By the definition of weight, $s$ can be written in the form $s=t_{1} \cdots t_{m} v_{0}$ where $v_{0}$ has weight greater than $c$ and the $t_{i}$ are of weight $c$ and are all simple commutators in $s_{1}, \cdots, s_{n}$. Then by the fundamental expansion formula ${ }^{5}$ for $(P Q \cdots)^{x}$ we have

$$
s^{q}=t_{1}^{q} \cdots t_{m}^{q} v_{0} v_{1}^{q} \cdots v_{j}^{q} w
$$

where $w \in F^{p}$ and the $v_{\beta}$ are all of weight greater than $c$. By definition $t_{\beta}^{q} \in \bar{H}_{q}$ and by our induction hypothesis $v_{\beta}^{q} \in \bar{H}_{q} \cup F^{p}$ and so $s^{q} \in \bar{H}_{q} \cup F^{p}$.

Corollary I. Let $s$ have weight $c$, for $c<p$. Then $s^{q} \in \bar{H}_{q} \cup F^{c+1}$.

Set $H_{q, c}=H_{q} \cap F^{c}$ and $\bar{H}_{q, c}=\bar{H}_{q} \cap F^{c}$. Then we have

Corollary II. For $c<p, H_{q, c} \cup F^{c+1}=\bar{H}_{q, c} \cup F^{c+1}$.

LEMMA II. $F_{q}^{c} / F_{q}^{c+1} \simeq F^{c} /\left(F^{c+1} \cup H_{q, c}\right)$.

We note first that applying the second homomorphism theorem ${ }^{6}$ to Hall's formula $F_{q}^{c}=\left(F^{c} \cup H_{q}\right) / H_{q}$ we obtain the result $F_{q}^{c}=F^{c} / H_{q, c}$ (for all $c$ ). Now

${ }^{5}$ See $[4$, formula 3.51$]$ or $[6$, p. 111$]$.

${ }^{6}$ See $[2$, p. 32].

${ }^{7}$ See $[4$, formula 2.491$]$ or $[2$, p. 119]. 


$$
\begin{aligned}
F^{c} /\left(F^{c+1} \cup H_{q, c}\right) & \simeq\left(F^{c} / H_{q, c}\right) /\left(\left[F^{c+1} \cup H_{q, c}\right] / H_{q, c}\right) \\
& \simeq F_{q}^{c} /\left(F^{c+1} /\left[H_{q, c} \cap F^{c+1}\right]\right)=F_{q}^{c} / F_{q}^{c+1},
\end{aligned}
$$

since $H_{q, c} \cap F^{c+1}=H_{q, c+1}$.

Set $Q_{q}^{c}=F_{q}^{c} / F_{q}^{c+1}$.

TheOREM I. For $c<p, Q_{q}^{c}$ is abelian of order $q^{N}, N=\psi_{c}(n)$.

Definition. $t_{1}, \cdots, t_{k}$ is said to be a basis for $F^{c} \bmod F^{c+1}$, if any operator $t$ of weight $c$ can be written uniquely in the form $t=\prod t_{i}^{d_{i}} \theta$ where $\theta \in F^{c+1}$.

Evidently such a basis exists, and by Witt's theorem ${ }^{8} k=N$; and we may choose the $t_{i}$ as simple commutators in the generators $s_{1}, \cdots, s_{n}$. Let $\rho_{i}$ be the image in $Q_{q}^{c}$ of $t_{i}$. Then since the $t_{i}$ are a basis for $F^{c} \bmod F^{c+1}$, any operator $\rho \in Q$ can be written in the form $\rho=\prod \rho_{i}^{d_{i}}$ where $0 \leqq d_{i}<q$. Hence the order of $Q_{q}^{c}$ is at most $q^{N}$ for any $c$. If the order of $Q_{q}^{c}$ is less than $q^{N}$ there exists a relation $\prod \rho_{i}^{d_{i}}=I$ where, say, $d_{j} \neq 0$.

If now $p>c$, this relation together with Corollary II and Lemma II imply that $\prod t_{i}^{d_{i}} \in \bar{H}_{q, c} \cup F^{c+1}$, or $\prod t_{i}^{d_{i}} \equiv \prod t_{t}^{q e_{i}} \bmod F^{c+1}$. Since the $t_{i}$ are a basis for $F^{c} \bmod F^{c+1}$ this requires $d_{i}-q e_{i}=0, i=1, \cdots, N$, which contradicts the assumption that $d_{j}$ and, therefore, $d_{j}-q e_{j}$ is not divisible by $q$. Hence there can be no relation between the $\rho_{i}$ and the theorem is proved.

Corollary III. For $p>c, G_{q, c}^{j}$ is of order $q^{m}$,

$$
m=\psi_{j}(n)+\cdots+\psi_{c}(n), \quad j=1, \cdots, c .
$$

4. Characteristic subgroups of $G=G_{p, c}$. A large variety of characteristic subgroups of $G$ can be obtained from the lower central series by sequences of joins, intersections, and commutations. In $G$ the upper and lower central series are identical; in particular, the central $C$ ( $=C_{p, c}$ ) of $G$ is $G^{p}$. The central quotient group of $G$ is $G_{p, c-1}$, and any characteristic subgroup $H$ of $G$ is mapped into a characteristic subgroup $H^{\prime}=H \cup C / C$ in $G_{p, c-1}$.

We say that $K$ is a minimal characteristic subgroup (m.c.s.) of $G$ if no proper subgroup of $K$ is characteristic in $G$. For $G=G_{p, c}$, every m.c.s. lies in the central. Indeed any normal subgroup of $G$ must contain commutators of weight $c$ and therefore must have an intersection not equal to $I$ with $C$. We turn now to the determination of all characteristic subgroups of $G$ which lie in $C$.

${ }^{8}$ See [7, Theorems 3 and 4, pp. 152-153]. 
Let $\bar{A}$ be any automorphism of $G$, and $H$ any characteristic subgroup of $G . \bar{A}$ induces an automorphism $\bar{A}(H)$ on $G / H$ and an automorphism $\bar{A}[H]$ on $H$. If in particular $H$ is $G^{2}$, the commutator subgroup of $G$, then $G / H$ is the abelian group of order $p^{n}$ and type $1,1,1, \cdots$. Let the generators of $G$ be $s_{i}, \cdots, s_{n}$, and let $t_{i}$ be the image in $G / G^{2}$ of $s_{i}$. Then $\bar{A}(H)$ takes the form $t_{i} \rightarrow t_{i}^{\prime}$ where

$$
t_{i}^{\prime}=\prod t_{j}^{a i j}, a_{i j} \in G F[p], \quad\left|a_{i j}\right| \neq 0 .
$$

Hence $\bar{A}$ itself must be of the form $s_{i} \rightarrow s_{i}^{\prime}$ where

$$
s_{i}^{\prime}=\prod s_{j}^{a_{i j}} r_{i}, \quad r_{i} \in G^{2} .
$$

To calculate $\bar{A}[C]$ we apply (5) with $k=c$. Since $G^{c+1}=I,(5)$ is now an equality and shows that $\bar{A}[C]$ is independent of the $r_{i}$. Indeed if we set $A=\left(a_{i j}\right)$ we see that the formal commutators $s_{i_{1}} \cdots i_{c}$ transform like tensors of rank $c$, that is, according to $A \times A \times \cdots \times A$ (Kronecker direct product with $c$ factors).

Denote by $M_{c}$ the whole space of tensors of rank $c$. It has dimension $n^{c}$. The group $A_{c}=\{A \times A \times \cdots \times A\}$ (c factors) is homomorphic to the group $\{A\}$ of linear transformations, and hence $M_{c}$ is a representation space for $\{A\}$. Brauer ${ }^{9}$ has proved the following theorem concerning the decompositions of this representation:

THEOREM II. If $K$ is a field of characteristic $p \neq 0$, the representation $A_{c}$ is completely reducible for $c<p$, and it splits into irreducible parts in exactly the same way as in the case of characteristic zero.

The mapping $x_{i_{1}} \ldots i_{c} \rightarrow s_{i_{1}} \cdots i_{c}$ (where of course products in $C$ are replaced by sums in $M_{c}$ ) establishes a homomorphic mapping of $M_{c}$ upon $C$ and this mapping is preserved under the group $A_{c}$, that is, $C$ is also a representation space for the group $A_{c}$. Let $\bar{C}$ denote $C$ written additively. Then $\bar{C}=M_{c}-W_{c}$, where $W_{c}$ contains all tensors whose image in $C$ is identity. We call $W_{c}$ the space of commutator relations, $W_{c}$ is evidently an invariant subspace of $M_{c}$ under the tensor group and by Theorem I it has dimension $n^{c}-\psi_{c}(n)$ if $p>c$. Because of the complete reducibility of the representation $A_{c}$ we can write $M_{c}=W_{c}+P_{c}$ where $P_{c}$ is likewise an invariant subspace of $M_{c}$, and furthermore the decomposition into irreducibly invariant subspaces of $P_{c}$ under $A_{c}$ will be the same as that of $C$ under the group of automorphisms of $G$. ( $\left(P_{c}\right.$ is not uniquely determined by $W_{c}$ but its decompositions are.) Let $R_{1}, \cdots, R_{t}$ be irreducibly invariant sub-

${ }^{9}$ See $[3$, p. 867] 
spaces of $M_{c}$ whose direct sum is $P_{c}$, and let $T_{1}, \cdots, T_{t}$ be the corresponding subgroups of $C$. Then the following theorem expresses the above arguments in group theoretic terms:

Theorem III. Any minimal characteristic subgroup is isomorphic to one of $T_{1}, \cdots, T_{t}$ and any characteristic subgroup $K$ of $G$ which lies in the central is the direct product of the minimal characteristic subgroups which it contains. ( $p>c$ is assumed throughout.)

The number of characteristic subgroups in $G$ is clearly independent of the number $n$ of generators provided that $n \geqq c$. Hence to obtain all characteristic subgroups of the set of groups $G_{p, c}$ with $p>c$ we need only consider those with $n=c$.

5. The groups $G_{p, 3}$ and $G_{p, 4}$. In this section we shall make use of the decomposition into irreducibly invariant subspaces of the tensor spaces $M_{3}$ and $M_{4}$. These can be readily obtained by a direct computation based upon the decomposition theorems of $M_{c}$ in general. ${ }^{10} \mathrm{We}$ suppose $n=3$ in $M_{3}$ and $n=4$ in $M_{4}$.

$M_{3}=\sum_{1}+\sum_{2,1}+\sum_{2,2}+\sum_{3}$ in which the summands have dimensions $10,8,8$ and 1 respectively. $W_{3}=\sum_{1}+\sum_{2,1}+\sum_{3}$ and hence $G_{p, 3}$ has just one m.c.s., its central.

$$
\begin{aligned}
M_{4}= & \sum_{1}+\sum_{2,1}+\sum_{2,2}+\sum_{2,3}+\sum_{3,1}+\sum_{3,2} \\
& +\sum_{4,1}+\sum_{4,2}+\sum_{4,3}+\sum_{5}
\end{aligned}
$$

in which the summands have dimensions $35,45,45,45,20,20,15$, 15,15 , and 1 respectively. $W_{4}=\sum_{1}+\sum_{2,1}+\sum_{2,2}+\sum_{3,1}+\sum_{3,2}+\sum_{4,1}$ $+\sum_{4,2}+\sum_{5}$ and hence $G_{p, 4}$ has two m.c.s., one of which is its second derived group. Let us denote these by $D$ and $E$.

$G_{p, 1}$ has no proper characteristic subgroups and the only proper characteristic subgroup of $G_{p, 2}$ is its central $G_{p, 2}^{2}$.

THEOREM IV. The only characteristic subgroups of $G_{p, 3}$ are the members of its lower central series.

Let $H$ be characteristic in $G_{p, 3}$. Then if $H \neq I$ or $C$, by Theorem III $H \supset C . H^{\prime}=H / C$ must then be $G_{p, 2}$ or its central. In the first case $H=G_{p, 3}$ and in the second case $H=G_{p, 3}^{2}$.

Theorem V. The only characteristic subgroups of $G_{p, 4}$ are $D, E$ and the members of the lower central series.

It is easy to see that if a characteristic subgroup $H \supset C$ then $H$ is in

${ }^{10}$ See for instance [1, Theorem $4.4 \mathrm{D}$, p. 129$]$. 
the lower central series. To complete the proof we show then that if $H \nsupseteq C, H=D$ or $E$. Since $H \nsupseteq C$, either $H^{\prime}=I$; in which case $H \subset C$ and therefore $H=D$ or $E$; or $H^{\prime} \supset G_{p, 3}^{3}$ (by Theorem IV). It remains now only to show that $H^{\prime} \supset G_{p, 3}^{3}$ implies $H \supset C$. If now $H^{\prime} \supset G_{p, 3}^{3}$, then $H \cup C \supset G_{p, 4}^{3}$ and hence for the commutator $s_{123}$ of weight 3 we have a factorization $s_{123}=h d$ where $h \in H$ and $d \in C$ (and so $d$ has weight not less than 4$)$. Since $H$ is normal $\left(h, s_{4}\right)=\left(s_{123} \cdot d^{-1}, s_{4}\right)=s_{1234} \in H$. But the conjugates of $s_{1234}$ generate $C$ so that $H \supset C$ contrary to hypothesis, and the theorem is proved.

For the sake of completeness we give a multiplication table for $G_{p, 3}$. Applying the formulas of $\$ 2$ and Theorem I we have for any operator $s$ of $G_{p, 3}$ a unique expression in the form

$$
s=s^{A}=\prod s_{i}^{a_{i}} \prod_{i<j} s_{i j}^{a_{i j}} \prod_{i \neq j} s_{i j j}^{a_{i j j}} \prod_{i<j<k} s_{i j k}^{a_{i j k} s_{j k i}^{a_{j k i}}} .
$$

If now $s^{C}=s^{A} s^{B}$, then applying the readily verified formula $\left(s_{1}^{\alpha}, s_{2}^{\beta}\right)$ $=s_{12}^{\alpha \beta} s_{121}^{\beta C} \alpha_{\alpha, 2} s_{122}^{\alpha C_{\beta, 2}}$ we obtain ${ }^{11}(i<j<k)$

$$
\begin{aligned}
c_{i} & =a_{i}+b_{i}, \quad c_{i j}=a_{i j}+b_{i j}-b_{i} a_{j}, \\
c_{i j j} & =a_{i j j}+b_{i j j}-b_{i} C_{a_{j}, 2}+b_{j} a_{i j}-b_{i} b_{j} a_{j}, \\
c_{j i i} & =a_{j i i}+b_{j i i}+a_{j} C_{b_{j}, 2}-b_{i} a_{i j}, \\
c_{i j k} & =a_{i j k}+b_{i j k}+b_{j} a_{i k}+b_{k} a_{i j}-b_{i} a_{j} a_{k}-b_{i} b_{j} a_{k}-b_{i} b_{k} a_{j}, \\
c_{j k i} & =a_{j k i}+b_{j k i}+b_{i} a_{j k}+b_{j} a_{i k}-b_{i} b_{j} a_{k} .
\end{aligned}
$$

\section{BIBLIOGRAPHY}

1. H. Weyl, The Classical Groups, Princeton University Press, 1939.

2. H. Zassenhaus, Lehrbuch der Gruppentheorie, Hamburger Mathematische Einzelschriften, vol. 21, 1937.

3. R. Brauer, Algebras connected with semi-simple groups, Annals of Mathematics, vol. 38 (1937), pp. 857-872.

4. P. Hall, Groups of prime power order, Proceedings of the London Mathematical Society, vol. 36 (1934), pp. 29-95.

5. Levi and van der Waerden, Über eine besondere Klasse von Gruppen, Abhandlungen aus dem mathematischen Seminar der Hamburgischen Universität, vol. 9 (1933), pp. 154-158.

6. W. Magnus, Über Beziehungen zwischen höheren Kommutatoren, Journal für die reine und angewandte Mathematik, vol. 177 (1937), pp. 105-115.

7. E. Witt, Treue Darstellung Liescher Ringe, Journal für die reine und angewandte Mathematik, vol. 177 (1937), pp. 152-160.

UNIVERSITY OF MichigAN

${ }^{11}$ For $p=3, s_{i j j}=s_{i i i}=I$ and $s_{i j k}=s_{j k i}$ so that (6) reduces to formula 9 of Levi and van der Waerden [5, p. 156]. 